\title{
A Framework of Nanomechanical Communication Systems Based on State Transitions
}

\author{
Ahmed O. Nasif \\ Dept. of Engineering Technology \\ University of Wisconsin-Oshkosh \\ Oshkosh, WI-54901 \\ $+1(920) 424-4159$ \\ nasif@uwosh.edu
}

\author{
Mohammad Upal Mahfuz \\ Natural and Applied Sciences \\ University of Wisconsin-Green Bay \\ Green Bay, WI-54311 \\ $+1(920) 465-2790$ \\ mahfuzm@uwgb.edu
}

\author{
Jagadeep Thota \\ Natural and Applied Sciences \\ University of Wisconsin-Green Bay \\ Green Bay, WI-54311 \\ $+1(920) 465-2817$ \\ thotaj@uwgb.edu
}

\begin{abstract}
In this paper, the potential of nanomechanical (NM) components to serve as a nanomechanical communication (NMC) system is explored in detail. In particular, a framework to identify the key aspects relating to the mechanical dynamics of a NM system with information transfer between a pair of communicating nanomachines. The proposed model is generic in the sense that it captures the motion of the NM components comprising of a NM system, such as nanoscale actuators, sensors, hinges, valves, gears, springs, etc., without referring to their specific structures, compositions or operational mechanisms. Such components are modeled by categorizing their motions into four types: (i) linearto-linear, (ii) linear-to-rotational, (iii) rotational-to-linear, and (iv) rotational-to-rotational. Current approach is to characterize the interface of any two nanomachines within a NM communication system. These two nanomachines can be adjacent (directly connected or coupled), or non-adjacent (indirectly connected or coupled) with other intervening NM components between them. These intervening NM components can also be nanomachines that work as relays in this case. The motion of a particular NM component is characterized by its motional degrees of freedom (MDOF), which is simply the number of discrete positions it can be in throughout its operation. Our model incorporates randomness due to thermal noise, ambient influences and other system uncertainties (like friction).
\end{abstract}

\section{CCS Concepts}

- Hardware Nanoelectromechanical systems

- Networks Physical links • Computer systems organization $\sim$ Sensors and actuators

\section{Keywords}

Nanomechanical systems; nanomachines; nanomechanical communications; channel modeling; state transition.

Permission to make digital or hard copies of all or part of this work for personal or classroom use is granted without fee provided that copies are not made or distributed for profit or commercial advantage and that copies bear this notice and the full citation on the first page. To copy otherwise, to republish, to post on servers or to redistribute to lists, requires prior specific permission and/or a fee. BICT 2017, March 15-16, Hoboken, United States

ISBN 978-1-63190-148-5

DOI: 10.4108/eai.22-3-2017.152410

Copyright $\odot 2017$ EAI techniques between a pair of nanomachines [1,2]. A nanomachine is a tiny machine of the size of nanoscale to microscale dimensions that has at least one dimension of up to $100 \mathrm{~nm} .{ }^{1}$ When size of machines goes down to nanoscale dimension, the properties of material changes. Individual nanomachines are extremely limited in terms of their functionalities and capabilities. As a result, there is a significant level of difficulty in scaling down the traditional macroscale communication systems to work at nanoscale dimensions. Therefore, there is a need for new communication techniques that would suit nanomachine communication between a pair of nanomachines and thus form a network of a huge number of nanomachines when they work collaboratively.

Communication between nanomachines can take place in several ways. In NMC, a pair of nanomachine are thought to be connected via mechanical hard junctions through which information is communicated from the transmitter to the receiver nanomachines [1]. Unlike other popular techniques of nanomachine communication, e.g. nano-electromagnetic (nano-EM) [3], molecular communication $[1,4]$, to the best of authors' knowledge, NMC is comparatively unexplored in the field of nanoscale communication systems mainly because it is sometimes considered infeasible to have hard-wired NM connections between nanomachines [1]. Nevertheless, to the best of authors' knowledge, for the first time ever, NMC as a communication means between a pair of nanomachines has been addressed in detail in this paper.

In traditional mechanical systems, independent infrastructure of electronic sensor and communication systems are deployed with ease, rendering the need of mechanical systems to serve as communication systems as well, unnecessary. In other words, traditional mechanical systems are very often electro-mechanical systems endowed with dedicated electronic communication capabilities.

In natural biological nanosystems, mechanical forces are used to convey information at the cellular level for regulating biochemical signaling and protein expression processes $[5,6]$. Similarly, we envision that the proposed NMC system can be used in engineered nanosystems to respond to mechanical stimuli and transfer information. For example, two nanorobots can communicate by coming into direct physical contact with each other, and then exchange information through mechanical motions. Repeated application of this process can allow information to be propagated

\footnotetext{
${ }^{1} 1$ nanometer $(\mathrm{nm})$ is a billion-th, i.e. $10^{-9}$, of a meter.
} 
over a larger region, the same way multihop communication is performed in wireless ad hoc networks.

In this paper, a framework of an NMC system is presented. Our main contribution is the modeling of the mechanical motions of an NM system using state transitions. We provide an expression for the transmission rate given in terms of number of symbols per movement for a simple NM transmitter-receiver pair, which depends on the minimum value of the motional degrees of freedom of the transmitter and the receiver.

\section{STRUCTURE OF AN NM SYSTEM}

In an NM system, a mechanical movement at one point in the system results in a corresponding desired movement at another point. When an NM system is being used as a communication system, a sequence of bits can be transferred from one point of the system to another through the propagation of a series of definite mechanical movements.

Our proposed model of NM communication system is generic in the sense that it captures the motion of the NM components, such as nanoscale actuators, sensors, hinges, valves, gears, springs, etc. comprising the NM system, without referring to their specific structures, compositions or operational mechanisms. We can view these families of components by categorizing their motions into four types: (i) linear-to-linear, (ii) linear-to-rotational, (iii) rotational-to-linear, and (iv) rotational-to-rotational. As a first study, we do not consider more sophisticated motion patterns. In particular, we characterize the interface between any two NM components within an NMC system. These two NM components can be adjacent, i.e., directly connected or coupled, or nonadjacent, i.e., indirectly connected or coupled with other intervening NM components in between.

\subsection{Motional Degrees of Freedom and Motional States}

We characterize the motion of a particular NM component by its motional degrees of freedom (MDOF), which is the total number of discrete rest positions it can be in throughout its operation. The arrival to a particular rest position of an NM component after executing a motion or movement is referred to as a motional state (MS). In other words, MDOF is simply the total number of possible MSs of an NM component. It is easy to find the MDOF of NM components that exhibit discrete motion or movement. For example, a nanoscale valve, which is either open or closed, has a MDOF of 2. Another example is a nanoscale gear with $n$ teeth, which has a MDOF of $n$.

What happens if the motions of an NM component do not take place in terms of discrete positions? That is, the NM system does not exhibit any steady-state distinct rest positions; its position is simply a continuously varying quantity. Nanoscale hinges, springs, etc. may exhibit such movement patterns. We model such 'analog' motion by 'digitizing' the motion into discrete position intervals or 'bins'. This is done in the same spirit as analog-todigital conversion performed in most electronic systems in use today: to leverage the advantages of digital representation, modeling and computing. For example, a smoothly varying continuous linear motion between two positions, $a$ and $b(b>a)$, can be represented by $n$ MDOFs, where each MS refers to a distinct uniform interval of width $(b-a) / n$. Similarly, a rotational motion between angles $\theta$ and $\varphi(\varphi>\theta)$ can be represented by $n$ MDOFs, with uniform angular intervals of $(\varphi-\theta) / n$. Of course, non-uniform intervals are also possible. We note that the choice of $n$ can be made to achieve a tradeoff between model accuracy and model complexity. We refer to this as the digitization of motions of an NM component that exhibits inherently continuous movements.

Hence, our model can represent discrete and continuous motions, as well as linear and rotational motions, by using the notion of MDOF, which allows us to encode mechanical MSs into transferrable information bits or symbols. In fact, as we shall see shortly, we will use this concept of MDOFs and MSs as our key modeling tool to explore communication capabilities of NM systems. We would like to explore the potential of NM systems to communicate in terms of the following two performance metrics: (A) symbol transmission rate, and (B) symbol error rate.

\section{NMC SYSTEM MODEL}

Suppose that two NM components are interfaced with each other directly, or indirectly through a number of intervening NM components. The types of motions involved could different combinations of the four types of motions mentioned earlier. The NM component triggering the motion is the transmitter (TX), and the NM component that is the intended target of that motion is the receiver ( $\mathrm{RX})$. The intervening NM components and interfaces between the TX and RX represent the channel. The time difference between the triggering of the motion at the TX until that motion is transferred to the $\mathrm{RX}$ is the propagation delay.

\subsection{Deterministic Model}

Consider first the scenario, where the system is assumed to be free of any kind of noise or undesired disturbances. In such a case, a particular movement at the TX results in a deterministic corresponding movement at the RX. This transfer of mechanical motions between two adjacent MN components can be leveraged to transfer information.

One way to accomplish this is by encoding all the possible MSs between the TX and RX into bits or symbols. For convenience, each MS can be represented as an MS index. As an example, a TX having 10 gear teeth may be represented by $10 \mathrm{MSs}$, with each MS index corresponding to a particular gear tooth position. Alternatively, the same gear could be represented by $5 \mathrm{MSs}$, with each MS index corresponding to a pair of particular and consecutive gear tooth positions. So, the encoding is simply a table, referred to as the MS transition table, which enlists all possible TX and RX MS transitions and the corresponding encoded information symbols (in binary representation) associated with those transitions.

In light of such an encoding between motion and information, we can interpret specific mechanical functions of an NM system as simply the transmission of specific bit strings propagated through various mechanical movements and maneuvers. We make the following assumptions regarding our model.

(i) An NM component can execute all of its possible MSs independently and in any sequence necessary for transmitting information.

(ii) Information is conveyed by the TX only by executing a mechanical motion starting from a particular MS and ending at another particular MS. As far as information transmission is concerned, we can ignore the detailed physics involved in the motion of the NM component transitioning from one rest position to another, or equivalently, from one MS to another.

(iii) From the perspective of end-to-end transfer of information, the intervening NM components between the TX and the RX do not really have a role in our model. Of course, in practical 
systems it may be appropriate to take into account the detailed properties and structures of the intervening elements.

(iv) An NM component may be capable of exhibiting only a limited number of pre-determined motion patterns. For an NM component to be able to convey information, it must have at least two MSs and the ability to move back and forth freely between them.

In order to represent or encode transitions between mechanical MSs into information symbols, a mapping between the MSs of the two NM components and the information symbols is necessary. Next, we demonstrate the encoding process using a few examples below.

We use the following notations throughout the paper.

Table 1: Notations

\begin{tabular}{|c|l|}
\hline Notation & \multicolumn{1}{|c|}{ Meaning } \\
\hline $\mathrm{C}=i \rightarrow j$ & $\begin{array}{l}\text { A particular MS transition from the initial state } i \\
\text { to the final state } j \text { of an NM component C }\end{array}$ \\
\hline$\left\{\mathrm{C}=i^{\prime} \rightarrow j\right\}$ & $\begin{array}{l}\text { A set of MS transitions comprising of all } \\
\text { possible initial states other than } i \text { to the final } \\
\text { state } j \text { of an NM component } \mathrm{C}\end{array}$ \\
\hline$\left\{\mathrm{C}=i \rightarrow j^{\prime}\right\}$ & $\begin{array}{l}\text { A set of MS transitions comprising of the initial } \\
\text { state } i \text { to all possible final states other than } j \text { of } \\
\text { an NM component C }\end{array}$ \\
\hline$\left\{\mathrm{C}=i^{\prime} \rightarrow j^{\prime}\right\}$ & $\begin{array}{l}\text { A set of MS transitions comprising of all } \\
\text { possible initial states other than } i \text { to all possible } \\
\text { final states other than } j \text { of an NM component C }\end{array}$ \\
\hline
\end{tabular}

Example 1: Consider a TX and an RX with MDOF of $N_{T X}=2$ and $N_{R X}=2$, respectively, with no feedback between the TX and RX. The corresponding MS transition table is shown below in Table 1. It is clear that there are four distinct MS transitions possible at the TX that result in four unique MS transitions at the RX. As a result, the TX can transmit $N_{T X} N_{R X}=2 \times 2=4$ symbols per MS transition to the RX.

Note that the self-transitions TX $=0 \rightarrow 0$ and TX $=1 \rightarrow 1$ are capable of conveying information since they represent possible motion patterns detectable at the RX. For example, in rotational motion, it represents a complete $360^{\circ}$ revolution. In linear motion, it represents some motion that starts and ends at the same location or location bin. Such cyclic motion can be used to transmit 1 bit of information per movement, where a ' 1 ' is conveyed through the periodic motion, and a ' 0 ' is conveyed by the absence of motion. However, a proper protocol between TX and RX needs to be established beforehand to distinguish between whether no motion represents a ' 0 ' or simply no communication. Pre-defined motion patterns can be used to identify the start and end of a communication session in the same way bit patterns or flags are used to identify the beginning and end of a frame in data link layer protocols [7].

Table 2: MS Transition Table of Example $1\left(N_{T X}=2, N_{R X}=2\right)$

\begin{tabular}{|c|c|c|}
\hline $\begin{array}{c}\text { MS Transitions } \\
\mathbf{T X}=\boldsymbol{i} \rightarrow \boldsymbol{j}\end{array}$ & $\begin{array}{c}\text { MS Transitions } \\
\mathbf{R X}=\boldsymbol{k} \rightarrow \boldsymbol{l}\end{array}$ & $\begin{array}{c}\text { Information } \\
\text { symbols }\end{array}$ \\
\hline $0 \rightarrow 0$ & $0 \rightarrow 0$ & 00 \\
\hline $0 \rightarrow 1$ & $0 \rightarrow 1$ & 01 \\
\hline $1 \rightarrow 0$ & $1 \rightarrow 0$ & 10 \\
\hline $1 \rightarrow 1$ & $1 \rightarrow 1$ & 11 \\
\hline
\end{tabular}

Example2: Consider a TX and an RX with MDOF of $N_{T X}=3$ and $N_{R X}=2$, respectively, with no feedback between the TX and RX. The corresponding MS transition table is shown below in Table 2 . As in Example 1, this system can convey 4 symbols per transition. This is because there are only 4 unique MS transitions available at the RX corresponding to the 9 unique MS transitions of the TX.

Table 3: MS Transition Table of Example $2\left(N_{T X}=3, N_{R X}=2\right)$

\begin{tabular}{|c|c|c|}
\hline $\begin{array}{c}\text { MS Transitions } \\
\mathbf{T X}=\boldsymbol{i} \rightarrow \boldsymbol{j}\end{array}$ & $\begin{array}{c}\text { MS Transitions } \\
\mathbf{R X}=\boldsymbol{k} \rightarrow \boldsymbol{l}\end{array}$ & $\begin{array}{c}\text { Information } \\
\text { symbols }\end{array}$ \\
\hline $0 \rightarrow 0$ & $0 \rightarrow 0$ & 00 \\
\hline $0 \rightarrow 1$ & $0 \rightarrow 1$ & 01 \\
\hline $0 \rightarrow 2$ & $1 \rightarrow 0$ & 10 \\
\hline $1 \rightarrow 0$ & $1 \rightarrow 1$ & 11 \\
\hline $1 \rightarrow 1$ & - & - \\
\hline $1 \rightarrow 2$ & - & - \\
\hline $2 \rightarrow 0$ & - & - \\
\hline $2 \rightarrow 1$ & - & - \\
\hline $2 \rightarrow 2$ & - & $\mathrm{r}$ \\
\hline
\end{tabular}

Example 3: Now, consider a TX and an RX with MDOF of $N_{T X}=$ 3 and $N_{R X}=3$, respectively, with no feedback between the TX and $\mathrm{RX}$. The corresponding MS transition table is shown below in Table 3. As expected, this system can convey 9 symbols per transition.

Table 4: MS Transition Table of Example $3\left(N_{T X}=3, N_{R X}=3\right)$

\begin{tabular}{|c|c|c|}
\hline $\begin{array}{c}\text { MS Transitions } \\
\mathbf{T X}=\boldsymbol{i} \rightarrow \boldsymbol{j}\end{array}$ & $\begin{array}{c}\text { MS Transitions } \\
\mathbf{R X}=\boldsymbol{k} \rightarrow \boldsymbol{l}\end{array}$ & $\begin{array}{c}\text { Information } \\
\text { symbols }\end{array}$ \\
\hline $0 \rightarrow 0$ & $0 \rightarrow 0$ & 000 \\
\hline $0 \rightarrow 1$ & $0 \rightarrow 1$ & 001 \\
\hline $0 \rightarrow 2$ & $0 \rightarrow 2$ & 010 \\
\hline $1 \rightarrow 0$ & $1 \rightarrow 0$ & 011 \\
\hline $1 \rightarrow 1$ & $1 \rightarrow 1$ & 100 \\
\hline $1 \rightarrow 2$ & $1 \rightarrow 2$ & 101 \\
\hline $2 \rightarrow 0$ & $2 \rightarrow 0$ & 110 \\
\hline $2 \rightarrow 1$ & $2 \rightarrow 1$ & 0000 \\
\hline $2 \rightarrow 2$ & $2 \rightarrow 2$ & 110 \\
\hline
\end{tabular}

Based on the above examples, we make the following observations.

a. The information transmission rate depends on the rate of the movements of the TX. Hence, we propose to quantify information transmission rate in terms of number of symbols per movement of the TX, instead of number of symbols per unit time. Of course, for particular NM systems, the movements can be converted into equivalent time durations yielding symbols per unit time specification of the transmission rate.

b. In the absence of any feedback between TX and RX, the $\mathrm{RX}$ can only receive information as allowed by its MDOF. In particular, an RX with $\mathrm{MDOF}=N_{R X}$ can at most receive $N_{R X}$ symbols per movement. Similarly, a TX with $\mathrm{MDOF}=N_{T X}$ can transmit at most $N_{T X}$ symbols per movement. As a consequence, a TX-RX pair can transfer at most 
$\left(\min \left\{N_{T X}, N_{R X}\right\}\right)^{2}$ symbols/movement.

Therefore, although the two NMC systems presented in Example 1 and Example 2 have different MDOFs, they have equal transmission rates.

c. To simplify the construction of the MS transition table, we can simply choose to make $\min \left\{N_{T X}, N_{R X}\right\}$ as the MDOF of both the TX and the RX. This can be done using the notion of digitization of MDOF mentioned earlier.

d. The simplicity of our model arises from its ability to skip the detailed structures and operations of the intervening NM components that are part of the channel, and characterize the MS transitions between the TX and the RX directly.

\subsection{Stochastic Model}

The effect of noise, friction and other types of external undesired influences on the NMC system is simply to cause MS transitions at the RX that are not specified in the MS transition table. For example, in Example 1, an error event occurs whenever the transition $\mathrm{TX}=0 \rightarrow 0$ results in $\mathrm{RX}=0 \rightarrow 1$ (see Table 1). All possible MS transitions for Example 1 are given below in Table 4. The transitions leading to error events are in blue and red.

Table 5: All possible MS transitions for Example1 $\left(N_{T X}=2\right.$, $N_{R X}=2$ ). (erroneous transitions are in blue and red)

\begin{tabular}{|c|c|c|c|}
\hline $\begin{array}{c}\text { MS } \\
\text { Transitions } \\
\mathbf{T X}=\boldsymbol{i} \rightarrow \boldsymbol{j}\end{array}$ & $\begin{array}{c}\text { MS } \\
\text { Transitions } \\
\mathbf{R X}=\boldsymbol{k} \rightarrow \boldsymbol{l}\end{array}$ & $\begin{array}{c}\text { Transmitted } \\
\text { Information } \\
\text { symbols }\end{array}$ & $\begin{array}{c}\text { Received } \\
\text { Information } \\
\text { symbols }\end{array}$ \\
\hline $0 \rightarrow 0$ & $0 \rightarrow 0$ & 00 & 00 \\
\hline $0 \rightarrow 0$ & $0 \rightarrow 1$ & 00 & 01 \\
\hline $0 \rightarrow 0$ & $1 \rightarrow 0$ & 00 & 10 \\
\hline $0 \rightarrow 0$ & $1 \rightarrow 1$ & 00 & 11 \\
\hline $0 \rightarrow 1$ & $0 \rightarrow 1$ & 01 & 01 \\
\hline $0 \rightarrow 1$ & $0 \rightarrow 0$ & 01 & 00 \\
\hline $0 \rightarrow 1$ & $1 \rightarrow 0$ & 01 & 10 \\
\hline $0 \rightarrow 1$ & $1 \rightarrow 1$ & 10 & 10 \\
\hline $1 \rightarrow 0$ & $1 \rightarrow 0$ & 10 & 11 \\
\hline $1 \rightarrow 0$ & $1 \rightarrow 1$ & 10 & 00 \\
\hline $1 \rightarrow 0$ & $0 \rightarrow 0$ & 10 & 01 \\
\hline $1 \rightarrow 0$ & $0 \rightarrow 1$ & 11 & 11 \\
\hline $1 \rightarrow 1$ & $1 \rightarrow 1$ & 11 & 10 \\
\hline $1 \rightarrow 1$ & $1 \rightarrow 0$ & 11 & 00 \\
\hline $1 \rightarrow 1$ & $0 \rightarrow 0$ & 11 & 01 \\
\hline $1 \rightarrow 1$ & $0 \rightarrow 1$ & 01 & 0 \\
\hline
\end{tabular}

In a stochastic formulation of our model, we assign probabilities to the erroneous MS transitions at the RX. The distribution of the probabilities captures the effect of noise and other physical disturbances on the RX. We observe that all erroneous transitions are not necessarily equally likely. Since the MSs represent locations, intuition suggests that under steady state operation, smaller location errors are likely to occur more frequently compared to larger location errors, especially for systems with large MDOFs.

As shown in Table 5, there are two types of erroneous transitions. The transitions in blue indicate where the initial state of the RX is correct but the final state is not. This type of error can happen even if the system transmits one symbol per movement and then resets itself before the next transmission. For example, given TX $=0 \rightarrow 0, \mathrm{RX}=0 \rightarrow 1$. The errors caused by the transitions shown in red may occur when there is no reset option available, and multiple symbols are transmitted back-to-back. For example, given $\mathrm{TX}=0 \rightarrow 0, \mathrm{RX}=1 \rightarrow 0$ or, $\mathrm{RX}=1 \rightarrow 1$.

From Table 5, we observe that for $N=2$, there are a total of 12 transitions that lead to error, which is given by $N^{2}\left(N^{2}-1\right)$. The first factor $N^{2}$ indicates the total number of TX-RX MS combinations. The second factor $\left(N^{2}-1\right)$ indicates the total number of erroneous transitions for each TX-RX MS combination. Their product gives the total number of erroneous transitions for a given value of $N$.

\section{CONCLUSION AND FUTURE WORK}

In this paper, a framework of an NMC system based on state transitions has been presented. A few future investigation threads have also been mentioned. It is possible for the RX to know the MSs of the TX by virtue of its mechanical coupling with the TX. In such a scenario, a higher transmission rate may be achievable. NMC schemes leveraging the knowledge of TX MSs, and feedback in general, are not considered in this paper, and will be explored in the future work of this research. Just as in traditional digital communications, intelligent transmission schemes can be devised by encoding multiple symbols together for transmission. Such schemes can allow for error detection and correction capabilities at the RX, and will be explored as part of the future work.

\section{REFERENCES}

[1] Akyildiz, I. F., Brunetti, F. and Blázquez, C., 2008. Nanonetworks: A new communication paradigm. Computer Networks, 52(12), pp.2260-2279.

[2] Bush, S. F. 2010. Nanoscale Communication Networks, Artech House.

[3] Jornet, J. M., and Akyildiz, I. F. 2010. Graphene-based nanoantennas for electromagnetic nanocommunications in the terahertz band. In Proceedings of the Fourth European Conference on Antennas and Propagation (pp. 1-5). IEEE.

[4] Mahfuz, M. U., Makrakis, D., and Mouftah, H. T. 2010. On the characterization of binary concentration-encoded molecular communication in nanonetworks, Nano Communication Networks, 1(4), 289-300.

[5] Freitas, R. A., 1999. Nanomedicine, volume I: basic capabilities (pp. 17-8). Georgetown, TX: Landes Bioscience.

[6] Vogel, V., 2010, January. Shrunk to nano: The secrete language of mechanical communication. In Micro Electro Mechanical Systems (MEMS), 2010 IEEE 23rd International Conference on (pp. 83-87). IEEE.

[7] Peterson, L. L., and Davie, B. S. 2012. Computer Networks: A Systems Approach, $5^{\text {th }}$ ed., Morgan Kaufmann, Burlington, MA. 